\title{
Differential contribution of leucocytes and spermatozoa to the generation of reactive oxygen species in the ejaculates of oligozoospermic patients and fertile donors
}

\author{
R. J. Aitken, D. Buckingham, K. West, F. C. Wu, K. Zikopoulos and \\ D. W. Richardson \\ MRC Reproductive Biology Unit, 37 Chalmers Street, Edinburgh EH3 9EW, UK
}

\begin{abstract}
Summary. Cells isolated from the ejaculates of a high proportion of patients exhibiting oligozoospermia are characterized by generation rates of reactive oxygen species that considerably exceed those obtained for the normal fertile population. The purpose of this study was to resolve the cellular source of this enhanced activity. Semen samples from a cohort of oligozoospermic patients and a group of fertile controls were fractionated on discontinuous Percoll gradients to generate three cell populations $(0,50$ and $100 \%$ ) of differing density. For each fraction, both the steady-state and the phorbolester-induced chemiluminescent signals were significantly $(P<0.001)$ greater for the oligozoospermic samples than for the fertile controls. In the fertile donors, leucocytes comprised the major source of reactive oxygen species, particularly in the low-density Percoll fractions; in oligozoospermic patients, however, spermatozoa were identified as a second major source of reactive oxygen species. Particularly striking was an intense phorbol-ester-induced chemiluminescent signal generated by oligozoospermic spermatozoa, purified by passage through isotonic Percoll and free of leucocyte contamination, which was 167 times greater than the median signal generated by the corresponding fraction from the fertile controls $(P<0.001)$. These results emphasize the importance of spermatozoa as a major source of reactive oxygen species in oligozoospermia and have implications for the diagnosis and treatment of this condition, as well as for the design of appropriate diagnostic strategies.
\end{abstract}

Keyords: oligozoospermia; reactive oxygen species; spermatozoa; human

\section{Introduction}

Despite the high incidence of male infertility (Hull et al., 1985), there are few effective methods for treating this condition. This paucity of therapeutic techniques stems from an underlying uncertainty concerning the mechanisms responsible for defective sperm function. Clearly, without a knowledge of these fundamental mechanisms there can be no progress on either the diagnosis or treatment of infertile males (Aitken, 1989).

An indication of at least one of the causes of male infertility has come from studies on the role of oxidative stress in compromising the functional competence of human spermatozoa. These cells are particularly susceptible to such stress in view of their high content of unsaturated fatty acids (Jones et al., 1979) and highly specialized capacity for generating reactive oxygen species (ROS), including superoxide anion and hydrogen peroxide (Aitken \& Clarkson, 1987a, b; Alvarez et al., 1987; Aitken et al., 1989). Moreover, a number of retrospective analyses have indicated that 
the excessive generation of ROS is associated with defective sperm function, particularly oligozoospermia (Aitken \& Clarkson, 1987; Aitken et al., 1989). Furthermore, in a recent prospective study (Aitken et al., 1991), the incidence of spontaneous pregnancy was shown to be negatively correlated with generation of ROS with approximately half of the oligozoospermic patients in the study population ( 8 of 17) exhibiting increased activity.

The source of the high levels of ROS observed in cases of male infertility is unresolved and clinically significant. The washed human ejaculate contains, in addition to spermatozoa, a variable number of leucocytes, particularly neutrophils (Wolff \& Anderson, 1988a, b; Aitken \& West, 1990; Barrett et al., 1990), which are powerful generators of ROS. If infiltrating leucocytes, rather than defective spermatozoa, are responsible for the oxidative stress observed in cases of male infertility, this will clearly have an impact on the therapeutic strategies that might be explored in such cases. In particular, the value of broad-spectrum antibiotic treatment could be assessed alongside the anti-oxidant regimens suggested on the basis of previous studies (Aitken \& Clarkson, 1988).

To determine the relative significance of leucocytes, spermatozoa and precursor germ cells in creating the oxidative stress associated with oligozoospermia, we have compared the generation of ROS in relation to the cellular composition of fractions isolated from the ejaculates of oligozoospermic human males and fertile donors.

\section{Materials and Methods}

\section{Sperm preparation}

The study population comprised a group of 17 oligozoospermic patients, who had produced semen specimens containing $<20 \times 10^{6}$ spermatozoa ml 1 on at least three occasions, and a group of 22 men of proven fertility who had fathered children within the past three years. The oligozoospermic ejaculates generated for the purpose of this study exhibited a median concentration of $10.4 \times 10^{6}$ spermatozoa ml ${ }^{-1}$ (range $\left.1-31\right)$; all but two $\left(29\right.$ and $31 \times 10^{6}$ spermatozoa $\mathrm{ml}$ ') of the samples were $<20 \times 10^{6} \mathrm{ml}$ '. All of the fertile donors produced semen specimens that conformed to the limits of normality set down by the World Health Organization (WHO, 1987).

The semen samples were produced by masturbation and collected into sterile containers for immediate transportation to the laboratory. After allowing at least $30 \mathrm{~min}$ for liquefaction to occur, the spermatozoa were fractionated on a discontinuous two-step Percoll gradient comprising $3 \mathrm{ml}$ isotonic Percoll overlaid with a further $3 \mathrm{ml} 50 \%$ Percoll in a $10 \mathrm{ml}$ conical-based sterile centrifuge tube. Isotonic Percoll was created by supplementing $10 \mathrm{ml}$ of $10 \times$ concentrated medium 199 (Flow Laboratories, Irvine, UK) with $300 \mathrm{mg}$ bovine serum albumin (BSA), $3 \mathrm{mg}$ sodium pyruvate and $0.37 \mathrm{ml}$ sodium lactate syrup, and adding $90 \mathrm{ml}$ of Percoll (Pharmacia, Uppsala, Sweden). This preparation was designated 100\% Percoll (Lessley \& Garner, 1983) and 50\% Percoll was prepared by dilution with an equal volume of medium BWW (Biggers et al., 1971).

One to $2 \mathrm{ml}$ of semen was layered on the top of each gradient and two or three individual gradients were used in the preparation of each semen sample. The gradients were centrifuged at $500 \mathrm{~g}$ for $20 \mathrm{~min}$ and the seminal plasma was then discarded and the cells collected into three fractions derived from (1) the semen-50\% Percoll interface $(0 \%$ fraction); (2) the $50-100 \%$ Percoll interface (50\% fraction); (3) the base of the $100 \%$ Percoll fraction ( $100 \%$ fraction). The cells from each fraction were resuspended in $7 \mathrm{ml}$ of BWW, centrifuged at $500 \mathrm{~g}$ for $5 \mathrm{~min}$ and finally resuspended at a sperm concentration of $2.5 \times 10^{6}$ spermatozoa $\mathrm{ml}$ '.

\section{Reactive oxygen species}

Reactive oxygen species were monitored by chemiluminescence (Aitken \& Clarkson, 1987a; Aitken et al., 1990), using 5-amino-2,3-dehydro-1,4 phthalazinedione (luminol) as the probe; $4 \mu \mathrm{l}$ of this probe, stored as a $25 \mathrm{~mm}$ stock solution in dimethyl sulfoxide, was added to $400 \mu \mathrm{l}$ of cell suspension and the chemiluminescent signal was measured on a Berthold LB 9505 luminometer. These measurements were made at a chamber temperature of $37^{\circ} \mathrm{C}$ over a total period of $30 \mathrm{~min}$. During the first $15 \mathrm{~min}$ of this time course, a $400 \mu \mathrm{l}$ aliquot of the cell suspension was incubated with $25 \mu \mathrm{mol} I$ ' luminol supplemented with $8.5 \mathrm{U}$ horseradish peroxidase (Type VI, $310 \mathrm{U} \mathrm{mg}^{-1}$ '; Sigma, St Louis, Mo) to sensitize the assay for the generation of extracellular hydrogen peroxide (Vilim \& Wilhelm, 1989; Aitken \& West, 1990). Once a steady-state signal had been established, the cells were stimulated by the addition of $100 \mathrm{~nm} 12-$ myristate, 13-acetate phorbol ester (PMA) and monitored for the following $15 \mathrm{~min}$. The chemiluminescent counts were integrated over two 5 min periods before and after the administration of PMA respectively. 


\section{Leucocytes}

The cells were pelleted at $500 \mathrm{~g}$ and resuspended in Dulbecco's phosphate-buffered saline (Flow Laboratories, Irvine, UK) at a concentration of $5 \times 10^{6} \mathrm{ml}^{-1} ; 5 \mu$ l of each cell suspension was dried down onto the wells of Hendley slides (C. A. Hendley, Essex, UK), wrapped in aluminium foil and stored at $-70^{\circ} \mathrm{C}$. Each Hendley slide contained a duplicate pair of positive control wells in which $5 \mu \mathrm{l}$ of a suspension of peripheral leucocytes had been air dried.

Before staining, the slides were allowed to reach room temperature and then fixed in acetone:methanol:formaldehyde (95:95:10) for $90 \mathrm{~s}$ and washed with Tris-buffered saline $(0.05 \mathrm{~mol}$ Tris I ', $0.15 \mathrm{~mol} \mathrm{NaCl} \mathrm{ml} \mathrm{',} \mathrm{pH} \mathrm{7.6).} \mathrm{Five} \mu$ l of a monoclonal antibody against the common leucocyte antigen (Scottish Antibodies Production Unit, Carluke, UK; S-149-201, cluster CD45), at a dilution of 1:10 was then spread evenly over each well. The slides were incubated for $30 \mathrm{~min}$ in a humidified chamber at room temperature before being washed twice in Tris-buffered saline.

The slides were subsequently incubated for $30 \mathrm{~min}$ with rabbit anti-mouse immunoglobulin (DAKO, High Wycombe, UK: Z259) diluted 1:25, washed twice with Tris-buffered saline and finally developed for $1 \mathrm{~h}$ with an alkaline-phosphatase-anti-alkaline-phosphatase complex (DAKO, High Wycombe, England; D 651) at a dilution of 1:50. Efficient labelling was then ensured by a repeat cycle of incubation with the alkaline-phosphatase-anti-alkalinephosphatase complex. Alkaline phosphatase activity was detected by incubating the slides for $18 \mathrm{~min}$ with a substrate containing naphthol As-MX phosphate $\left(0.5 \mathrm{mmol}^{-1}\right) 2 \%$ dimethylformamide, $0.01 \mathrm{mmol}$ levamisole $1^{\prime}$ and $3.9 \mathrm{mmol} 1$ 'Fast Red TR in $0.1 \mathrm{~mol} 1$ ' Tris, $\mathrm{pH} 8.2$. The slides were finally counterstained with haematoxylin and mounted in Apathy's aqueous mounting medium. For scoring purposes, $5 \mu$ l aliquots of each sperm suspension were evenly spread over an area defined by the individual wells of a 12-well Hendley slide (C. A. Hendley, Essex, UK). From a knowledge of the volume equivalent of each high-powered field and the concentration of spermatozoa in the suspension, the number of leucocytes per $10^{7}$ spermatozoa could be ascertained after at least 30 fields had been counted. The analysis was carried out in duplicate; the minimal number of leucocytes that could be detected with this method was $0.02 \times 10^{4}$ per $10^{7}$ spermatozoa. Nucleated round cells that did not stain with the monoclonal antibody and exhibited the appropriate morphology (WHO, 1987) were scored as precursor germ cells.

\section{Statistical analysis}

The frequency distribution of the data was examined and transformations $(\log , \log x+1)$ used for normalization where appropriate. The $\log (x+1)$ transformation was used for variables such as leucocyte counts, which included zero values, since the log of ' 0 ' cannot be found. Graphical data are presented in the form of box plots in which the 10 , $25,50,75$ and 90 th percentiles are indicated. Linear regression analysis and paired or unpaired $t$ tests were performed on the transformed data using the STATVIEW and STATWORKS programmes.

\section{Results}

\section{Reactive oxygen species}

$100 \%$ Percoll fraction. The $100 \%$ Percoll fraction gave a median (10th-90th percentile) basal chemiluminescent response of $3.12 \times 10^{4}\left(1.73-39.82 \times 10^{4}\right)$ counts per $5 \mathrm{~min}$ in the fertile donors, increasing significantly $(P<0.001)$ to $12.57 \times 10^{4}\left(2.34-323.48 \times 10^{4}\right)$ counts per 5 min after stimulation with the phorbol ester, PMA (Fig. 1). The $100 \%$ fraction from the oligozoospermic patients exhibited a median basal generation rate of ROS of $25.4 \times 10^{4}\left(4.91-1169.48 \times 10^{4}\right)$ counts per $5 \mathrm{~min}$, which was considerably $(P<0.001)$ greater than the basal chemiluminescent signal recorded from the fertile donors (Fig. 1). Furthermore, addition of PMA to these oligozoospermic sperm suspensions led to a large $(P<0.001)$ increase in the chemiluminescent signal to $1431.00 \times 10^{4}\left(98.86-80812.00 \times 10^{4}\right)$ counts per $5 \mathrm{~min}$, which was more than 100 times greater $(P<0.001)$ than the PMA response recorded in the fertile controls (Fig. 1). The percentage of recovered spermatozoa that partitioned into the isotonic Percoll fraction was significantly $(P<0.05)$ lower in the oligozoospermic patients than the fertile donors, giving median (10th-90th percentiles) values of $14 \cdot 5 \%(4 \cdot 9-28 \cdot 4 \%)$ and $26 \cdot 2 \%(3 \cdot 6-39 \cdot 2 \%)$, respectively. However, the proportion of spermatozoa recovered in the $100 \%$ Percoll fraction did not correlate with the generation of ROS in either group of subjects, in the presence or absence of PMA.

$50 \%$ Percoll fraction. The basal chemiluminescent signal given by the cells isolated on the $50-100 \%$ Percoll interface from the fertile ejaculates exhibited a median value of $9 \cdot 72 \times 10^{4}(1 \cdot 41-$ $156 \cdot 16 \times 10^{4}$ ) counts per 5 min. After PMA stimulation, a sudden burst of ROS production 


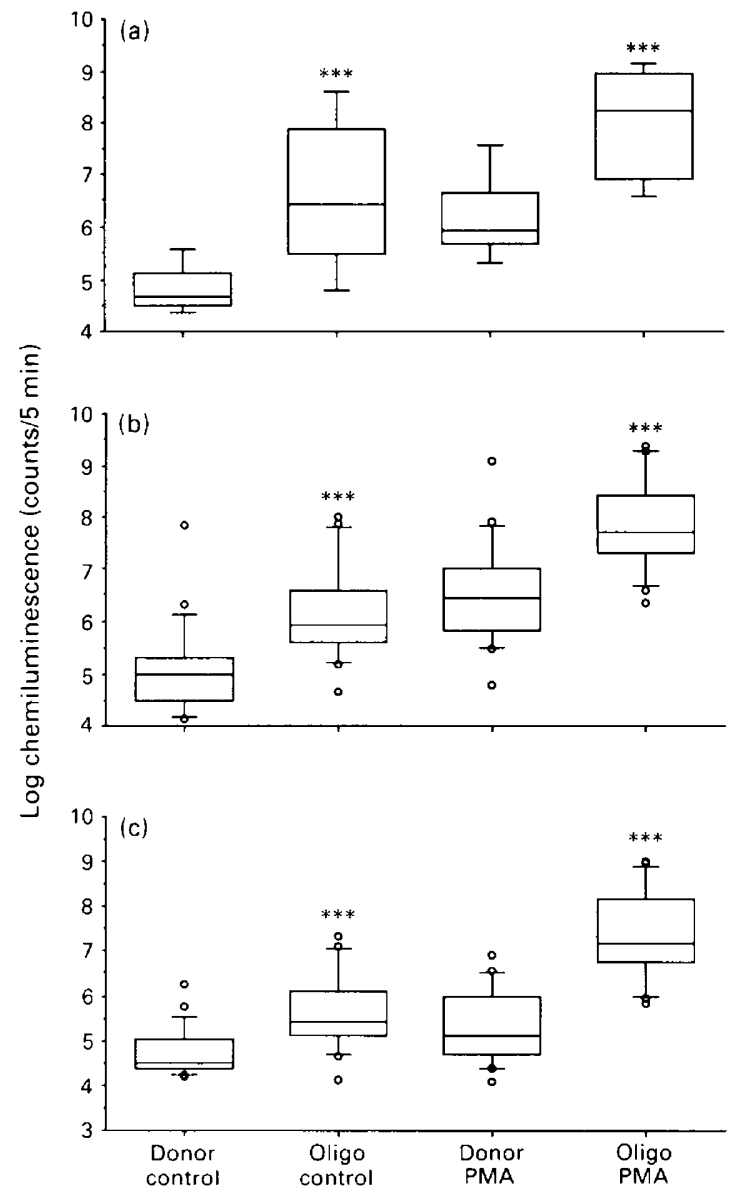

Fig. 1. Box plots of generation of ROS in the three cellular fractions isolated at (a) the semenPercoll interface (b) the 50-100\% Percoll interface and (c) the base of the $100 \%$ Percoll layer. Boxes indicate 25 and 75 percentiles while the horizontal line within the box indicates the position of the median. Vertical lines give 10 and 90 percentiles while single data points indicate extreme values outside these limits. ${ }^{* * *} P<0.001$. PMA: 12-myristate, 13 -acetate phorbol ester; control: treatment with vehicle alone; Donor: fertile donors; Oligo: oligozoospermic patients.

occurred, raising $(P<0 \cdot 001)$ the chemiluminescent counts to $280.80 \times 10^{4}\left(32 \cdot 22-6940.60 \times 10^{4}\right)$ counts per $5 \mathrm{~min}$, which was significantly $(P<0.01)$ greater than the PMA response observed with the corresponding $100 \%$ fractions.

The cell populations isolated on the $50-100 \%$ Percoll interface following centrifugation of the oligozoospermic samples generated a high basal median count of $85.72 \times 10^{4}(15.92-$ $\left.1479.00 \times 10^{4}\right)$ counts per $5 \mathrm{~min}$, which was significantly greater $(P<0.001)$ than the corresponding fraction from the fertile donors. Furthermore, addition of PMA to these sperm suspensions resulted in a rapid, significant $(P<0.001)$ rise in the chemiluminescent signal to a median value of $5255.00 \times 10^{4}\left(524.28-186980.00 \times 10^{4}\right)$ counts per $5 \mathrm{~min}$, which represented a 30 -fold increase $(P<0.001)$ over the PMA-induced signal obtained with the corresponding cell fraction from the fertile controls (Fig. 1).

The median (10th-90th percentiles) percentage of recovered spermatozoa that partitioned into the $50-100 \%$ Percoll interface was not significantly different in the oligozoospermic $(54 \cdot 3 \%$ : 
$15 \cdot 5-75 \cdot 9 \%$ ) and fertile donor (51.9\%: $31 \cdot 3-70 \cdot 9 \%)$ populations and did not correlate with the generation rate of ROS in the presence or absence of PMA.

$0 \%$ Percoll fraction. The cells that failed to penetrate the $50 \%$ Percoll layer (the $0 \%$ fraction) gave a median chemiluminescent signal of $4.46 \times 10^{4}\left(2 \cdot 36-38.30 \times 10^{4}\right)$ counts per $5 \mathrm{~min}$ in the fertile donors, increasing $(P<0.001)$ to $83.71 \times 10^{4}\left(19.77-3725.00 \times 10^{4}\right)$ counts per 5 min after the addition of PMA. This response to phorbol ester stimulation by the $0 \%$ fractions was significantly $(P<0.01)$ greater than the PMA-induced signals obtained from the cells isolated at the base of the isotonic (100\%) Percoll fraction from the same ejaculates (Fig. 1).

The low-density, $0 \%$, fractions obtained from the oligozoospermic patients generated intense basal chemiluminescent signals, which were significantly greater than those from the $100 \%$ fractions from the same ejaculates $(P<0.01)$ and the corresponding fraction from the fertile donors $(P<0.001)$, giving a median signal of $264.90 \times 10^{4}\left(6.20-38570.00 \times 10^{4}\right)$ counts per $5 \mathrm{~min}$. After the addition of PMA, the median signal increased $(P<0.001)$ to $17340 \times 10^{4}(376 \cdot 16-$ $\left.145520 \times 10^{4}\right)$ counts per 5 min, a rate which was more than 200 -fold $(P<0.001)$ greater than the corresponding fraction from the fertile donors (Fig. 1).

The median (10th-90th percentiles) percentage of recovered spermatozoa that partitioned into the $0-50 \%$ Percoll interface was not significantly different in the oligozoospermic $(32.7 \%: 14.9-$ $78 \cdot 3 \%)$ and fertile donor $(16 \cdot 0 \%: 5 \cdot 0-64 \cdot 1 \%)$ populations and did not correlate with the generation rate of ROS in the presence or absence of PMA.

In summary these chemiluminescent data have served to emphasize that, despite the considerable differences between individuals in the generation of reactive oxygen species (Fig. 1), the oligozoospermic samples consistently exhibited significantly higher generation rates of reactive oxygen species compared with the fertile controls, both in the steady-state situation and after stimulation with PMA. Furthermore, although there was a tendency for the highest chemiluminescent counts to derive from the low density $(0 \%$ and $50 \%)$ Percoll fractions in both groups of subjects, the significant enhancement of generation of ROS associated with oligozoospermia was observed in all three Percoll fractions, including the highly purified (see below) sperm populations pelleting in isotonic (100\%) Percoll.

\section{Cell composition}

Leucocytes. The presence of leucocytes in the various Percoll fractions was carefully monitored using immunocytochemical techniques to determine whether the intense chemiluminescence observed in cases of oligozoospermia was a consequence of leucocytic infiltration (Fig. 2).

In both the fertile donors and the oligozoospermic patients there were significantly fewer leucocytes in the high-density, $100 \%$, Percoll fractions than in the $50 \%$ fractions $(P<0.05)$ or $0 \%$ $(P<0.001)$ fractions. Moreover the oligozoospermic samples contained significantly $(P<0.01)$ more leucocytes than the fertile donors when the $0 \%$ and $50 \%$ fractions were considered, giving median values (10th-90th percentiles) of $8.39 \times 10^{4}\left(0.63-224.00 \times 10^{4}\right)$ and $2.75 \times 10^{4}(0.00$ $130.40 \times 10^{4}$ ) leucocytes per $10^{7}$ spermatozoa respectively for these fractions in the oligozoospermic patients, compared with corresponding values of $0.35 \times 10^{4}\left(0.00-3.09 \times 10^{4}\right)$ and $0.02 \times 10^{4}$ $\left(0 \cdot 00-5 \cdot 77 \times 10^{4}\right)$ leucocytes per $10^{7}$ spermatozoa in the fertile controls. In contrast, the median concentration of leucocytes in the $100 \%$ Percoll fractions was not significantly different in the oligozoospermic patients and fertile donors, giving median values (10th-90th percentiles) of $0.11 \times 10^{4}\left(0.00-18.39 \times 10^{4}\right)$ and $0.00 \times 10^{4}\left(0 \cdot 00-0 \cdot 11 \times 10^{4}\right)$ leucocytes per $10^{7}$ spermatozoa for these groups respectively (Fig. 2), despite the considerable differences in generation of ROS (Fig. 1).

The elevated concentration of leucocytes recorded in the low density Percoll fractions isolated from the oligozoospermic patients should not be taken as evidence of leucocytic infiltration into these ejaculates, but rather as a reflection of the low numbers of spermatozoa characteristic of this condition. In this study the leucocyte concentrations were expressed per $10^{7}$ spermatozoa to give an indication of the degree of leucocyte contamination of the sperm suspensions used for the analysis 

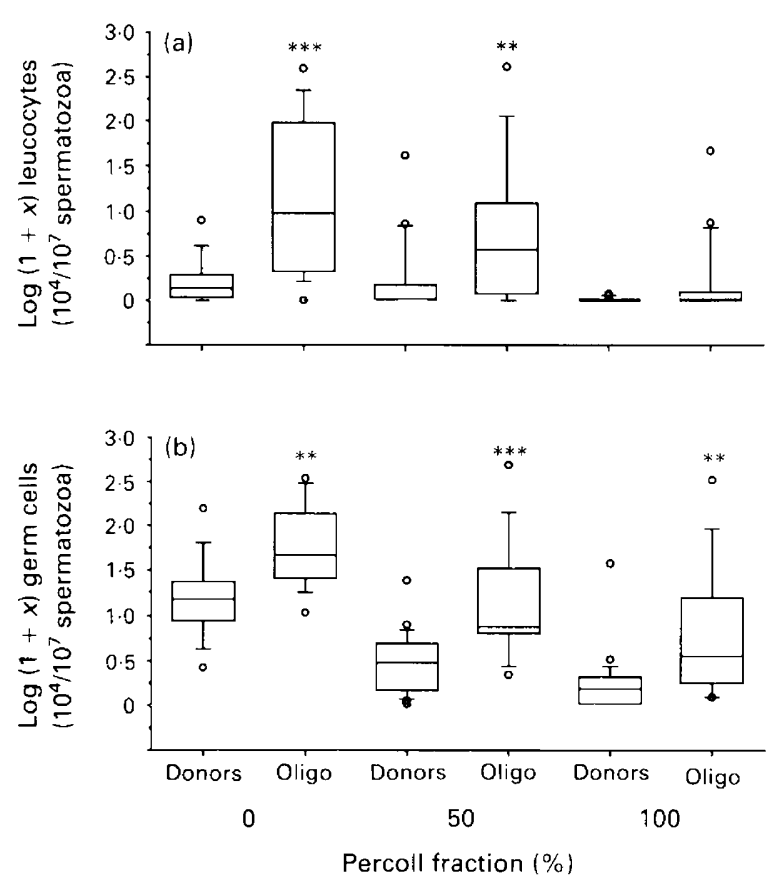

Fig. 2. Box plots of the concentration of (a) leucocytes and (b) precursor germ cells in the three Percoll fractions isolated from the ejaculates of fertile donors (Donors) and oligozoospermic patients (Oligo). Boxes indicate 25 and 75 percentiles while the horizontal line within the box indicates the position of the median. Vertical lines give 10 and 90 percentiles and single data points indicate extreme values outside these limits. ${ }^{* * *} P<0.001,{ }^{* *} P<0.01 .0 \%$ : interface between semen and 50\% Percoll; 50\%: interface between 50\% and 100\% Percoll; $100 \%$ : cells pelleted in isotonic Percoll.

of the generation of ROS. An obvious consequence of this notation system was that as numbers of spermatozoa declined, as in the case of the oligozoospermic patients, the ratio of leucocytes: spermatozoa increased even where the degree of leucocytic infiltration into the ejaculate was the same as in the fertile controls.

The total number of leucocytes present in each ejaculate was calculated to determine whether the oligozoospermic condition was, in fact, associated with an enhanced leucocytic infiltration into the seminal compartment. Summation of the leucocyte numbers recovered from the individual Percoll fractions gave a higher median (10th-90th percentiles) value for the oligozoospermic samples of $35.59 \times 10^{4}\left(6.50-1395.01 \times 10^{4}\right)$ leucocytes per ejaculate, than for the fertile controls, $19.62 \times 10^{4}\left(0.00-206.5 \times 10^{4}\right)$ leucocytes per ejaculate. However, examination of the raw data (Fig. 3) revealed considerable overlap in the leucocyte counts obtained for these two groups of subjects, such that the group means for the transformed data were not significantly different.

Clinically, the significant finding was that only one out of 17 oligozoospermic patients exhibited a leucocyte concentration $\left(13.54 \times 10^{6}\right.$ leucocytes $\left.\mathrm{ml}^{-1}\right)$ exceeding the normal fertile range recorded in this study and the $1 \times 10^{6} \mathrm{ml}^{-1}$ threshold recommended by the WHO (1987).

Precursor germ cells. The situation with respect to the presence of precursor germ cells in the ejaculates was broadly similar to that recorded for seminal leucocytes. Hence there was a significant $(P<0.05)$ tendency for these cells to concentrate in the low density, $0 \%$ portion of the Percoll gradients, and their concentration was significantly $(P<0.01)$ higher in the oligozoospermic samples than in the fertile controls, for all three Percoll fractions (Fig. 2). However, computation of 


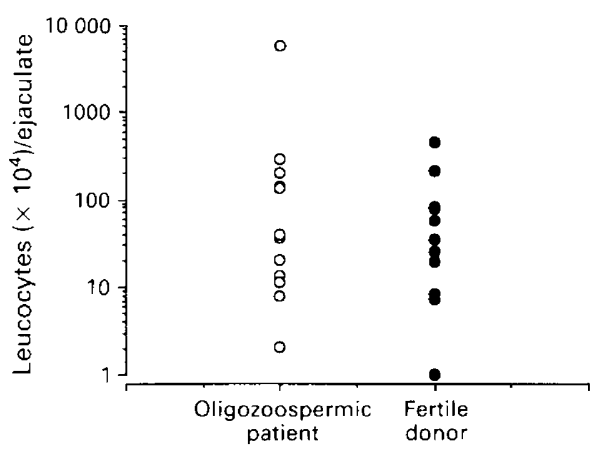

Fig. 3. Total number of leucocytes per ejaculate in the oligozoospermic patients $(O)$ and fertile controls (๑).

the total number of germ cells in the ejaculates from each group of subjects failed to reveal any significant difference between the oligozoospermic population and the fertile controls.

\section{Relationship between cell composition and ROS}

$0 \%$ fraction. The high steady-state generation rate of ROS characteristic of this fraction was not significantly correlated with the concentration of leucocytes or precursor germ cells in either group of subjects. After stimulation with PMA, however, a significant correlation with leucocyte concentration did emerge, in both the oligozoospermic patients $(P<0.001 ; r=0.787)$ and the fertile donors $(P<0.05 ; r=0.546)$, as shown in Fig. 4.

$50 \%$ fraction. The association between the generation of ROS and the presence of leucocytes was even more apparent in the $50 \%$ Percoll fractions. Scatterplot analysis of this data set revealed the presence of a skewed distribution, due to the presence of a disproportionate number of samples with leucocyte counts approximating to zero, which could not be corrected by log transformation (Fig. 4). This problem was resolved by dividing the data set into its two principal components. When the analysis focused on only those samples containing leucocytes, the log-transformed data were found to approximate to a normal distribution and linear regression analysis could be undertaken. These analyses revealed an extremely close relationship between the chemiluminescent counts and the degree of leucocytic infiltration $(P<0.001, r=>0.8)$, in both the donor and oligozoospermic populations, regardless of whether the basal or PMA-induced signals were considered (Fig. 5).

A second component of this data set comprised a distinct cohort of samples, including both patients and donors, in which leucocytes were completely absent and yet the intensity of the chemiluminescent signals varied by $2-3 \log$ orders of magnitude (Fig. 4). This variation presumably reflected differences in the ability of the spermatozoa to generate ROS, since no significant relationship was observed between this activity and the concentration of the only other cell type present in these $50 \%$ Percoll fractions, i.e. precursor germ cells.

$100 \%$ fraction. In contrast, within the $100 \%$ Percoll fractions, a significant correlation $(P<0.05 ; r=0.591)$ was observed between the basal, steady-state generation rates of ROS and the presence of precursor germ cells in the oligozoospermic samples, but not in the fertile donors.

The leucocyte concentrations in the $100 \%$ fractions were, again, so highly skewed towards zero that meaningful linear regression analysis was not possible. Examination of the scattergrams indicated that on the infrequent occasions when leucocytes did penetrate into this Percoll layer, the chemiluminescent counts were consistently high (Fig. 4), three (17.6\%) of the oligozoospermic samples clearly being associated with high leucocyte counts. The subsequent analyses focused on 


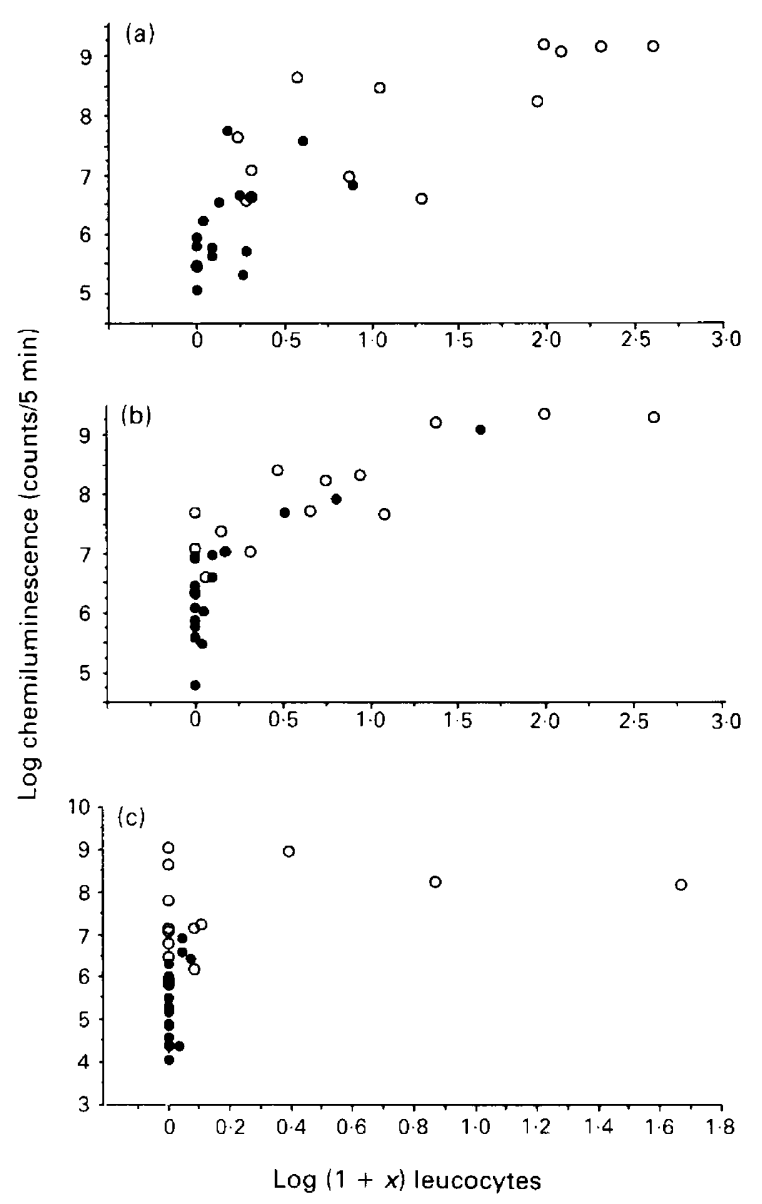

Fig. 4. Relationship between the 12-myristate, 13-acetate phorbol ester (PMA)-induced chemiluminescent signals and the concentration of leucocytes for (a) the $0 \%$, (b) $50 \%$ and (c) $100 \%$ Percoll fractions in fertile donors $(\bullet)$ and oligozoospermic patients $(O)$.

only those samples from which leucocytes were absent to circumvent this problem of leucocytic contamination (Fig. 6).

The median chemiluminescent signal generated by purified sperm suspensions prepared from the fertile donors was $2.36 \times 10^{4}\left(1.68-11.39 \times 10^{4}\right)$ counts per $5 \mathrm{~min}$, increasing $(P<0.001)$ to $7 \cdot 76 \times 10^{4}\left(2 \cdot 21-110.53 \times 10^{4}\right)$ counts per $5 \mathrm{~min}$ after the addition of phorbol ester (Fig. 6$)$. The steady-state chemiluminescent signal generated by the oligozoospermic specimens, in the absence of leucocyte contamination, was a log order of magnitude greater $(P<0.001)$ than the fertile controls, giving a median signal of $27.38 \times 10^{4}\left(2 \cdot 80-990.20 \times 10^{4}\right)$ counts per $5 \mathrm{~min}$. After exposure to PMA this signal increased $(P<0.001)$ still further to a median value of $1290.50 \times 10^{4}$ $\left(75.91-72810.00 \times 10^{4}\right)$ counts per $5 \mathrm{~min}$, eight out of $10(80 \%)$ oligozoospermic samples exhibiting PMA-induced chemiluminescence signals above the normal range (Fig. 6).

The intensity of the median oligozoospermic chemiluminescent signal corresponded to a $167-$ fold increase over the response given by purified donor spermatozoa to PMA stimulation. The limited overlap between the PMA-induced chemiluminescent signals generated by these purified sperm suspensions obtained from the oligozoospermic patients and fertile controls underlined the potential of such measurements in the diagnosis of male infertility. 
(a)

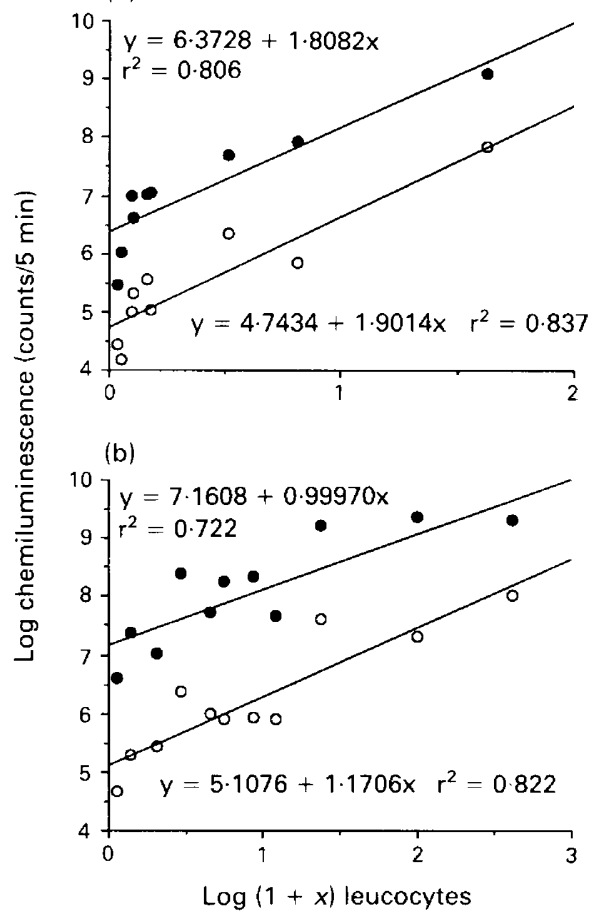

Fig. 5. Relationship between leucocyte concentrations and ROS for cell populations isolated on the $50-100 \%$ Percoll interface in which leucocytes were detected in (a) human fertile donors and (b) oligozoospermic patients. Basal chemiluminescent signal $(O) ; 12$-myristate, 13-acetate phorbol ester (PMA)-induced response $(\bullet) . P<0.001$ for all regression lines.

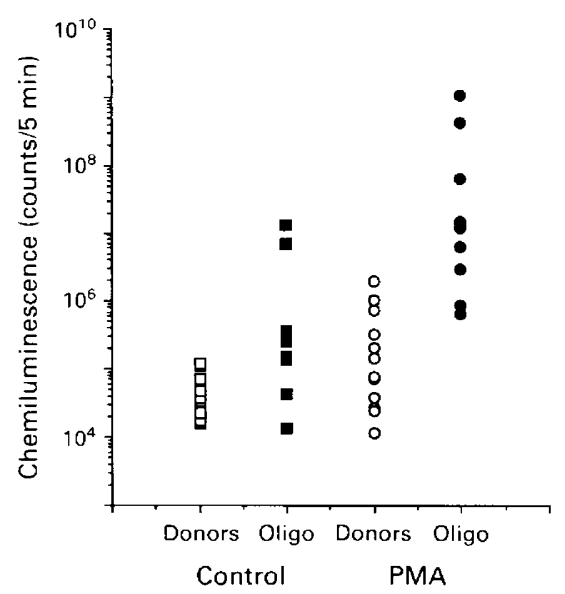

Fig. 6. Chemiluminescent signals for sperm suspensions isolated on $100 \%$ isotonic Percoll and free of leucocyte contamination in fertile donors (Donors) and oligozoospermic patients (Oligo). Baseline signals given by fertile donors $(\square)$ and oligozoospermic patients $(\boldsymbol{D})$ in the presence of vehicle alone; responses to 12-myristate, 13-acetate phorbol ester (PMA) given by fertile donors $(O)$ and oligozoospermic patients $(\mathbf{O})$. 


\section{Discussion}

Fractionation of the normal human ejaculate on discontinuous Percoll gradients leads to the isolation of highly motile, functionally competent spermatozoa in the high-density region of such gradients, which are characterized by a low capacity for generation of ROS (Aitken \& Clarkson, 1988). Conversely, the cells isolated in the low-density regions of these Percoll gradients are characterized by diminished motility and a poor capacity for spermatozoa-oocyte fusion, in association with a significant increase in the production of reactive oxygen species (Aitken \& Clarkson, 1988). Recent evidence suggests that there are two potential sources of oxygen radicals in the low-density Percoll fractions, comprising infiltrating leucocytes and the spermatozoa themselves (Aitken \& West, 1990). Determination of the origin of ROS in the human ejaculate is important, since this information will have a significant impact on the therapeutic strategies used to treat cases in which oxidative stress appears to play a causative role.

An example of a free-radical-associated male pathology is oligozoospermia (Aitken et al., 1989); approximately $50 \%$ of such cases are associated with generation rates of ROS above the normal fertile range. Here we have addressed the cellular origin of ROS in cases of oligozoospermia to determine the relative importance of leucocytic infiltration and aberrant sperm metabolism in creating the oxidative stress associated with this condition.

The oligozoospermic ejaculates were associated with highly significant increases in the generation rate of ROS in all three Percoll fractions, relative to the fertile controls. To some extent this increase was associated with higher concentrations of leucocytes in the oligozoospermic specimens, which exceeded the concentrations obtained for the fertile population for each Percoll fraction. This was not a reflection of leucocytic infiltration into the oligozoospermic ejaculates but rather an artefact, created by the method used to express the leucocyte concentration (as a ratio of leucocytes to spermatozoa) and the need to concentrate the oligozoospermic samples to achieve the sperm concentrations required to conduct the chemiluminescence measurements. Clearly, as the spermatozoa are concentrated, the concentration of any contaminating cell type, such as leucocytes, will also be increased.

In addition to ROS originating from seminal leucocytes, this study has also produced clear evidence for the generation of these powerful oxidants by the spermatozoa themselves. Furthermore, the major difference between the oligozoospermic specimens and the fertile controls lay in the relative contribution of the spermatozoa to the oxidant generating capacity of the ejaculate. This was clearly demonstrated in the $100 \%$ Percoll fractions isolated from the oligozoospermic patients that were free of leucocytic contamination and yet exhibited chemiluminescent signals $>150$ times greater than those recorded in normal fertile samples after stimulation with PMA (Fig. 6).

The validity of this conclusion depends to some extent on the accuracy and sensitivity of the leucocyte counting procedures used. If the mean number of leucocytes in a sperm suspension is low, there is a probability, defined by Poisson distribution theory, that a proportion of randomly-selected aliquots will be devoid of these cells, and the sample erroneously characterized as leucocytefree. However, it is extremely unlikely that such sampling errors could account for the enhanced chemiluminescent signals observed in the oligozoospermic patients because the same source of error would apply to the fertile samples, in which the generation rate of ROS was consistently low.

If spermatozoa are the major source of ROS in the oligozoospermic samples, there are clear implications for the use of Percoll gradients as a preparative technique in assisted reproduction procedures such as in vitro fertilization. In cases where defective sperm function is associated with the excessive generation of ROS by the spermatozoa, it is probable that even the cells isolated in isotonic Percoll will be associated with peroxidative damage.

Care, however, must be exercised in interpreting data on the production of ROS by sperm populations. In the absence of techniques to resolve the generation of ROS at the cellular level, it is 
impossible to determine the size of the sperm population actively engaged in this activity. Clearly, if there is heterogeneity within the sperm population with respect to the generation of ROS, opportunities will exist for interceding with anti-oxidants in vitro (Aitken \& Clarkson, 1988) that might not be realized if the entire sperm population is involved in excessive oxidant generation.

These findings therefore confirm the conclusions of an earlier study (Aitken et al., 1989) in that they reveal that a high proportion of oligozoospermic specimens are characterized by the excessive generation of ROS, a major source of which is the spermatozoa themselves. In terms of the incidence of this pathology, eight of the ten oligozoospermic samples purified on isotonic Percoll and found to be free from contaminating leucocytes exhibited chemiluminescent counts above the normal fertile range. These preliminary data will obviously need to be substantiated with larger numbers of patients if the true incidence of infertility involving oxidative stress created by defective spermatozoa is to be established.

Although it is not known when during spermatogenesis the excessive generation of oxidants is initiated, the fact that the ROS originate from the spermatozoa is probably a significant aspect of the pathology. A germ cell source implies that ample time is available for the ROS to damage both the free radical generating spermatozoa themselves and any normal spermatozoa in the immediate vicinity. Furthermore, once the process of lipid peroxidation has been initiated, the selfpropagating nature of this process would ensure a progressive spread of the damage throughout the sperm population.

There was some evidence in this study of a relationship between the presence of precursor germ cells and the generation of ROS by the oligozoospermic specimens purified on $100 \%$ isotonic Percoll. If this is confirmed it would suggest that the enhanced generation of ROS observed in cases of oligozoospermia is initiated sometime before spermiogenesis. The absence of similar correlations in the low-density Percoll fractions may have been due to the confounding effects of leucocytes or the possibility that precursor cells recovered in the $0 \%$ or $50 \%$ regions of Percoll gradients are not viable.

The pathological condition observed in oligozoospermic patients, involving spermatozoa as the predominant source of ROS, must be clearly differentiated from the situation pertaining in the normal fertile population. Hence, although the ejaculates provided by normal fertile donors occasionally generate intense chemiluminescent signals with luminol (Aitken et al., 1989), this study has shown that such activity is invariably associated with the infiltration of leucocytes. The damaging effect of ROS originating from leucocytes may be moderated if the site of infiltration is confined to the prostate or seminal vesicles. Under such circumstances, the first contact between the spermatozoa and leucocyte-derived free radicals would be at the moment of ejaculation when the spermatozoa would be protected by the powerful antioxidant factors in seminal plasma (Jones et al., 1979).

The chemiluminescent signals generated by human spermatozoa have been shown to have prognostic value in long-term prospective studies involving untreated couples with a normal female partner (Aitken et al., 1991). The results obtained in the present study indicate that accurate interpretation of such chemiluminescence data requires the identification of the cellular source of the oxidative stress. In this case, a rational refinement of the chemiluminescent technique for the diagnosis of male fertility would be to use isotonic Percoll to generate purified sperm suspensions, free from leucocyte contamination, for chemiluminescence analyses based on the stimulation of spermatozoa with phorbol ester (Fig. 6). If a simple luminometer is available, such assessments could be readily incorporated into a routine seminology laboratory and used to screen all patients, not just those exhibiting oligozoospermia.

We gratefully acknowledge the assistance of $\mathrm{H}$. Bowie and D. Harkiss in the preparation of spermatozoa for this study. 


\section{References}

Aitken, R.J. (1989) The role of free oxygen radicals and sperm function. Int. J. Androl. 12, 95-97.

Aitken, R.J. \& Clarkson, J.S. (1987a) Generation of reactive oxygen species by human spermatozoa. In Free Radicals: Recent Developments in Lipid Chemistry, Experimental Pathology and Medicine, pp. 333-335. Eds T. Dormandy \& C. Rice-Evans. Richelieu Press, London.

Aitken, R.J. \& Clarkson, J.S. (1987b) Cellular basis of defective sperm function and its association with the genesis of reactive oxygen species by human spermatozoa. J. Reprod. Fert. 81, 459-469.

Aitken, R.J. \& Clarkson, J.S. (1988) Significance of reactive oxygen species and anti-oxidants in defining the efficacy of sperm preparation techniques. J. Androl. 9, 367-376.

Aitken, R.J. \& West, K.M. (1990) Analysis of the relationship between reactive oxygen species production and leucocyte infiltration in fractions of human semen separated on Percoll gradients. Int. J. Androl. 13, 433-451.

Aitken, R.J., Clarkson, J.S., Hargreave, T.B., Irvine, D.S. \& Wu, F.C.W. (1989) Analysis of the relationship between defective sperm function and the generation of reactive oxygen species in cases of oligozoospermia. J. Androl. 10, 214-220.

Aitken, R.J., Clarkson, J.S. \& Fishel, S. (1990) Generation of reactive oxygen species, lipid peroxidation and human sperm function. Biol. Reprod. 41, 183-187.

Aitken, R.J., Irvine, D.S. \& Wu, F.C. (1991) Prospective analysis of sperm oocyte fusion and reactive oxygen species generation as criteria for the diagnosis of infertility. Am. J. Obstet. Gynec. 164, 542-551.

Alvarez, J.G., Touchstone, J.C., Blasco, L. \& Storey, B.T. (1987) Spontaneous lipid peroxidation and production of hydrogen peroxide and superoxide in human spermatozoa. $J$. Androl. 8, 338-348.
Barratt, C.L.R., Bolton, A.E. \& Cooke, I.D. (1990) Functional significance of white blood cells in the male and female reproductive tract. Hum. Reprod. 5, 639-644.

Biggers, J.S., Whitten, W.K. \& Whittingham, D.G. (1971) The culture of mouse embryos in vitro. In Methods in Mammalian Embryology, pp. 86-116. Ed. J. C. Daniel Jr. Freeman, San Francisco.

Hull, M.G.R., Glazener, C.M.A., Kelly, N.J., Conway, D.I., Foster, P.A., Hunton, R.A., Coulson, C., Lambert, P.A., Watt, E.M. \& Desai, K.M. (1985) Population study of causes, treatment and outcome of infertility. Br. Med. J. 291, 1693-1697.

Jones, R., Mann, T. \& Sherins, R. (1979) Peroxidative breakdown of phospholipids by human spermatozoa, spermicidal properties of fatty acid peroxides and protective action of seminal plasma. Fert. Steril. 31, $531-537$.

Lessley, B.A. \& Garner, D.L. (1983) Isolation of motile spermatozoa by density gradient centrifugation in Percoll. Gamete Res. 7, 49-61.

Vilim, V. \& Wilhelm, J. (1989) What do we measure by a luminol dependent chemiluminescence of phagocytes? Free Rad. Biol. Med. 6, 623-628.

Wolf, H. \& Anderson, D.J. (1988a) Immunohistologic characterization and quantitation of leukocyte subpopulations in human semen. Fert. Steril.49,497-504.

Wolff, H. \& Anderson, D.J. (1988b) Evaluation of granulocyte elastase as a seminal plasma marker for leukocytospermia. Fert. Steril. 50, 129-132.

World Health Organization (1987) WHO Laboratory Manual for the Examination of Human Semen and Semen-Cervical Mucus Interaction. Cambridge University Press, Cambridge.

Received 20 February 1991 